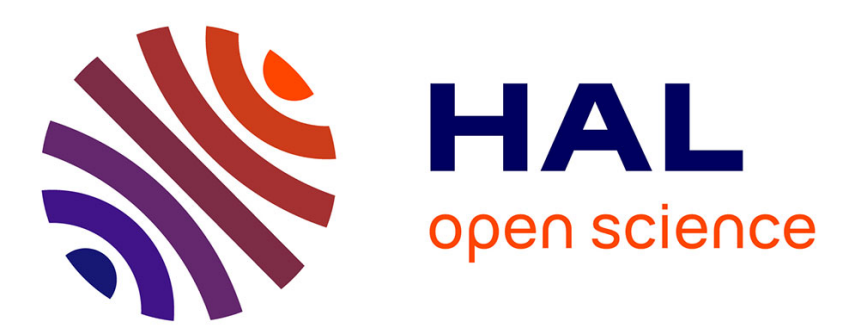

\title{
Synthesis, resolution, and determination of absolute configuration of protected $\alpha$-ethynylphenylalanine enantiomers
}

Z Benfodda, David Bénimèlis, Marion Jean, Jean-Valère Naubron, Valerie Rolland, Patrick Meffre

\section{To cite this version:}

Z Benfodda, David Bénimèlis, Marion Jean, Jean-Valère Naubron, Valerie Rolland, et al.. Synthesis, resolution, and determination of absolute configuration of protected $\alpha$-ethynylphenylalanine enantiomers. Amino Acids, 2015, 47 (5), pp.899-907. 10.1007/s00726-015-1917-1 . hal-02002634

\section{HAL Id: hal-02002634 \\ https://hal.science/hal-02002634}

Submitted on 11 Feb 2019

HAL is a multi-disciplinary open access archive for the deposit and dissemination of scientific research documents, whether they are published or not. The documents may come from teaching and research institutions in France or abroad, or from public or private research centers.
L'archive ouverte pluridisciplinaire HAL, est destinée au dépôt et à la diffusion de documents scientifiques de niveau recherche, publiés ou non, émanant des établissements d'enseignement et de recherche français ou étrangers, des laboratoires publics ou privés. 


\title{
Synthesis, resolution, and determination of absolute configuration of protected $\alpha$-ethynylphenylalanine enantiomers
}

\author{
Z. Benfodda $\cdot$ D. Bénimélis $\cdot$ M. Jean $\cdot$ J.-V. Naubron $\cdot$ \\ V. Rolland $\cdot$ P. Meffre
}

\begin{abstract}
Racemic-protected $\alpha$-ethynylphenylalanine was synthesized from DL-2-benzylserine using $\alpha$-benzylserinal as key intermediate and was successfully resolved by HPLC on a chiral stationary phase at a semipreparative scale. The absolute configuration of both enantiomers was determined by vibrational circular dichroism.
\end{abstract}

Keywords $\alpha$-Ethynylphenylalanine $\cdot$ Quaternary amino acids · Unsaturated amino acid - Chiral HPLC resolution . Absolute configuration

\section{Introduction}

$\alpha$-Alkyl $\alpha$-amino acids constitute an interesting class of non-proteinogenic amino acids and play a crucial role in the

Z. Benfodda $(\varangle) \cdot$ D. Bénimélis $\cdot$ P. Meffre

UNIV. NIMES, EA7352 CHROME, Rue du Dr G. Salan,

30021 Nîmes Cedex 1, France

e-mail: zohra.benfodda@unimes.fr

Z. Benfodda $\cdot$ D. Bénimélis $\cdot$ V. Rolland $\cdot$ P. Meffre IBMM, UMR-CNRS5247, Universités Montpellier 1 and 2, Place Eugène Bataillon, 34095 Montpellier, France

M. Jean

Aix Marseille Université, Centrale Marseille, CNRS, ISm2 UMR

7313, 13397 Marseille, France

J.-V. Naubron

Spectropole, Aix Marseille Université, 13397 Marseille Cedex

20, France development of peptides and peptidomimetics as therapeutics agents (Avenoza et al. 2001; Cativiela and Diaz de Villegas 2000, 2007; Hruby et al. 1997; Olma et al. 2012; Sawyer 1997). The additional alkyl substituent could prevent the free rotation of the residue side chain leading to unique folding when incorporated into peptides. They can severely restrict the conformations of the peptide backbone and provide key informations concerning the conformation responsible for biological recognition (Venkatraman et al. 2001; Tanaka 2007). Peptides containing quaternary $\alpha$-amino acids also tend to have increased hydrophobicity, as well as an increased stability toward both chemical (O'Connor and Liu 2003; Polinelli et al. 1992) and metabolic decompositions (Almond et al. 1962; Khosla et al. 1981).

Unsaturated $\alpha$-amino acids have turned out to be especially important building blocks for incorporation in protein structure due to the diverse reactivities of the multiple bonds and their ability to introduce biologically active functionalities (Bouifraden et al. 1999; Rutjes et al. 2000). These compounds are often used in peptide chemistry to confer $\beta$-turn secondary structure to induce new properties (Duggan et al. 2005).

Furthemore, several unsaturated amino acids were used as enzyme inhibitors (Tolman and Sedmera 1988; Keith et al. 1975; Rando 1974; Metcalf and Jund 1977; Berkowitz et al. 2004) in particular $\beta, \gamma$-unsaturated amino acid derivatives: $\alpha$-vinyl glycine is known to inhibit pyridoxal phosphate dependant enzymes such as amino acid decarboxylase (Maycock et al. 1979; Danzin et al. 1981; Tendler et al. 1987; Berkowitz et al. 2001), $\alpha$-ethynyl glycine is a well-known natural antibiotic and is a suicide substrate for alanine racemase (Walsh 1979; Kuroda et al. 1980a; Kuroda 1980b; Meffre and LeGoffic 1996).

Finally, with the advent of the "click chemistry concept", an amplified interest in acetylenic amino acids can 
be expected (Kaiser et al. 2005; Johansson and Pedersen 2012).

Despite the interest of these $\beta, \gamma$-unsaturated $\alpha$-alkyl amino acid derivatives, few syntheses have been described in the literature. In this field, the synthesis of both enantiomers of $\alpha$-ethynylalanine (Avenoza et al. 1999a; Colson and Hegedus 1993), the racemic and enantiomerically pure synthesis of $\alpha$-ethynyl- $\beta, \beta, \beta$-trifluoroalaninates (Shchetnikov et al. 2010; Crucianelly et al. 2004), and the racemic synthesis of $\alpha$-ethynyltyrosine and their derivatives (Patchett and Taub 1980; Taub and Patchett 1977) have been reported.

In this paper, we focus our attention particulary on the synthesis of $\alpha$-ethynylphenylalanine. To the best of our knowledge, only three methods for the synthesis of the racemic $\alpha$-ethynylphenylalanine backbone (as free or protected form) have been described in the literature. One of them (Casara and Metcalf 1978) claimed the synthesis of the fully protected $\alpha$-ethynylphenylalanine as well as the free amino acid but with no experimental details nor detailed analysis. Recent papers (Gonzalez et al. 2010; Finkbeiner et al. 2014) report the synthesis of racemic $\mathrm{N}, \mathrm{O}$ protected $\alpha$-ethynylphenylalanine and show the interest and the difficulties to synthesize a $\beta, \gamma$-alkynyl- $\alpha$-amino acid. Gonzalez and co-workers described the direct alkynylation of 2-nitro-3-phenylpropanoate using the hypervalent iodine reagent TMS-ethynyl-1,2-benziodoxol-3(1H)-one as electrophilic alkyne source. They obtained the N,O-protected $\alpha$-ethynylphenylalanine in three steps (48\% over yield) starting from 2-nitro-3-phenylpropanoate. Finkbeiner and co-workers developed a direct electrophilic alkynylation of azlactones (which are accessible from amino acids via cyclodehydration) by using alkynyl(phenyl)iodonium salts. The ring-opening of the corresponding azlactone with $\mathrm{KF} /$ $\mathrm{MeOH}$ gave the $\mathrm{N}, \mathrm{O}$-protected $\alpha$-ethynylphenylalanine in one step (98\% yield). However this compound has not been described in enantiomerically pure forms yet.

$\beta, \gamma$-unsaturated $\alpha$-alkyl amino acid synthesis represents a synthetic challenge (Di Giacomo et al. 2008). Therefore as a part of ongoing studies to look for new constrained amino acid in our laboratory (Ortial et al. 2014), we describe here a convenient synthesis of racemic protected $\alpha$-ethynylphenylalanine, the separation of two enantiomers by chiral HPLC ready for peptide synthesis and the determination of their absolute configuration.

\section{Materials and methods}

\section{General}

All reagents were purchased from the Aldrich Chemical Co. and used without any purification. Moisture sensitive reactions were carried out under dry nitrogen. Solvents were distilled from the appropriate drying agents immediately prior to use. NMR spectra were recorded with a Brucker Avance 300 spectrometer (300 and $75 \mathrm{MHz}$ for ${ }^{1} \mathrm{H}$ and ${ }^{13} \mathrm{C}$ NMR, respectively), a Bruker Avance 400 spectrometer (400 and $100 \mathrm{MHz}$ for ${ }^{1} \mathrm{H}$ and ${ }^{13} \mathrm{C}$ NMR, respectively); the chemical shifts are given in ppm relative to $\mathrm{Me}_{4} \mathrm{Si}$ for the ${ }^{1} \mathrm{H}$, and ${ }^{13} \mathrm{C}$ as internal standard. Data are reported as follows: chemical shifts, multiplicity ( $\mathrm{s}=$ singlet, $\mathrm{d}=$ doublet, $\mathrm{t}=$ triplet, $\mathrm{q}=$ quartet, $\mathrm{br}=$ broad, $\mathrm{dd}=$ double doublet, $\mathrm{dt}=$ double of triplet, $\mathrm{m}=$ multiplet), integration, coupling constants (Hz). Low-resolution electrospray ionization (ESI) mass was recorded on a micromass Platform Electrospray mass spectrometer. Spectra were recorded in positive mode $\left(\mathrm{ESI}^{+}\right)$. High resolution mass spectra (HRMS) were obtained by electrospray using a TOF analyzer Platform.

IR spectra were obtained using a Jasco FT-IR 410 instrument $\left(\nu \mathrm{cm}^{-1}\right)$ as a thin film on $\mathrm{NaCl}$ disc as stated. Reactions were monitored with Merck Kieselgel $60 \mathrm{~F}_{254}$ precoated aluminium silica gel plates $(0.25 \mathrm{~mm}$ thickness $)$ using ninhydrine as visualizing agent. Purifications by column chromatography were performed using silica gel 60 (230-400 mesh). Melting points were determined on a Stuart scientific SMP3 apparatus and are uncorrected. Nitrogen inversion in the oxazolidine ring or slow interconversion of both amide or carbamate conformers of compounds 3, 4, 5, 6 causes considerable line broadening and duplication of signals in the ${ }^{1} \mathrm{H}$ and ${ }^{13} \mathrm{C}$ NMR spectra.

Synthesis of DL-2-[(tertbutoxycarbonyl)amino]2-benzylserine (1)

DL- $\alpha$-benzylserine $(10 \mathrm{~g}, 51 \mathrm{mmol})$ and tetramethylammonium hydroxyde pentahydrate $(9.28 \mathrm{~g}, 51 \mathrm{mmol})$ were added to freshly distilled acetonitrile $(500 \mathrm{~mL})$. The mixture was stirred at room temperature until a solution had formed and $\mathrm{Boc}_{2} \mathrm{O}(16.7 \mathrm{~g}, 77 \mathrm{mmol})$ was then added, and stirring was continued for $48 \mathrm{~h}$. A further portion of $\mathrm{Boc}_{2} \mathrm{O}(5.59 \mathrm{~g}$, $26 \mathrm{mmol}$ ) was added and the mixture was stirred for an additionnal $24 \mathrm{~h}$ so at which point TLC analysis indicates complete formation of $\mathbf{1}$. The solvent was removed in vacuo and the residue was partitioned between water $(80 \mathrm{~mL})$ and ethyl acetate $(80 \mathrm{~mL})$. The aqueous layer was then acidified with solid citric acid to $\mathrm{pH} 3-4$. The aqueous solution was extracted three times with ethyl acetate and the combined organic extracts were washed with water, dried with $\mathrm{Na}_{2} \mathrm{SO}_{4}$, filtered, and concentrated under reduced pressure to give compound $\mathbf{1}$ as white solid $(15.06 \mathrm{~g}, 100 \%)$.

IR ( $\mathrm{NaCl}): 3,489\left(v_{\mathrm{OH}}\right), 3,350\left(v_{\mathrm{NH}}\right), 3,014\left(v_{\mathrm{CHAr}}\right), 1,685$ $\left(v_{\mathrm{C}=\text { Oacid }}\right)$; mp: $139{ }^{\circ} \mathrm{C} ;{ }^{1} \mathrm{H}$ NMR $\left(300.13 \mathrm{MHz}, \mathrm{CDCl}_{3}\right)$ : $1.38\left[\mathrm{~s}, 9 \mathrm{H}, \mathrm{C}\left(\mathrm{CH}_{3}\right)_{3}\right], 3.08,3.28\left(2 \mathrm{~d}, 2 \mathrm{H},{ }^{2} \mathrm{~J}=14 \mathrm{~Hz}\right.$, $\left.\mathrm{CH}_{2} \mathrm{Ph}\right), 3.9,4.13\left(2 \mathrm{~d}, 2 \mathrm{H},{ }^{2} \mathrm{~J}=12 \mathrm{~Hz}, \mathrm{CH}_{2} \mathrm{OH}\right), 5.33$ (s, $1 \mathrm{H}, \mathrm{NH}), 7.07,7.18\left(2 \mathrm{~m}, 5 \mathrm{H}, \mathbf{H}_{\mathrm{Ar}}\right), 6.9$ (br, $\left.1 \mathrm{H}, \mathrm{OH}\right) ;{ }^{13} \mathrm{C}$ 
$\operatorname{NMR}\left(75 \mathrm{MHz}, \mathrm{CDCl}_{3}\right): 28.3\left[\left(\mathrm{CH}_{3}\right)_{3}\right], 37.3\left(\mathrm{CH}_{2} \mathrm{Ph}\right), 65.5$ $\left(\mathbf{C H}_{2} \mathrm{OH}\right), 65.8\left[\mathbf{C}\left(\mathrm{CH}_{2} \mathrm{Ph}\right)\right], 80.6\left[\mathbf{C}\left(\mathrm{CH}_{3}\right)_{3}\right], 127.2\left(\mathbf{C}_{\mathrm{HAr}}\right)$, $128.5\left(2 \mathbf{C}_{\mathrm{HAr}}\right), 129.9\left(2 \mathbf{C}_{\mathrm{HAr}}\right), 134.9\left(\mathbf{C}_{\mathrm{Ar}}\right), 155.7\left(\mathrm{NCO}_{2}\right)$, $174.8\left(\mathrm{CO}_{2} \mathrm{H}\right)$.

Synthesis of (RS)-2-(tertbutoxycarbonylamino)2-benzylserine methyl ester(2)

To a cold solution (ice-water bath) of $\mathbf{1}(15 \mathrm{~g}, 50.8 \mathrm{mmol})$ in dimethylformamide $(70 \mathrm{~mL})$ was addedsolid potassium carbonate $(8.42 \mathrm{~g}, 60.9 \mathrm{mmol})$. After stirring for $10 \mathrm{~min}$ in an ice-water bath, methyl iodide $(6.32 \mathrm{~mL}, 101.6 \mathrm{mmol})$ was added to the white suspension and stirring continued at $0{ }^{\circ} \mathrm{C}$ for $30 \mathrm{~min}$ whereupon the mixture solidifies. The reaction was warmed to room temperature and stirred for an additonal hour or so at which point TLC analysis indicates complete formation of the methyl ester. The reaction mixture was filtered by suction, and the filtrate partitioned between ethyl acetate $(40 \mathrm{~mL})$ and water $(40 \mathrm{~mL})$. The organic layer was washed with brine, dried with $\mathrm{Na}_{2} \mathrm{SO}_{4}$, filtered, and concentrated in vacuo to give compound $\mathbf{2}$ as a pale amber oil which was used without any further purification $(15.47 \mathrm{~g}, 98 \%)$.

IR $(\mathrm{NaCl}): 3,434\left(v_{\mathrm{OH}}\right.$ et $\left.v_{\mathrm{NH}}\right), 2,980\left(v_{\mathrm{CHAr}}\right), 1,714$ $\left(v_{\mathrm{C}=\text { Oester }}\right), 1,698\left(v_{\mathrm{C}=\text { Ocarbamate }}\right) ;{ }^{1} \mathrm{H}$ NMR $(400.13 \mathrm{MHz}$, $\left.\mathrm{CDCl}_{3}\right): 1.47\left[\mathrm{~s}, 9 \mathrm{H}, \mathrm{C}\left(\mathrm{CH}_{3}\right)_{3}\right], 3.1$ and $3.39(2 \mathrm{~d}, 2 \mathrm{H}$, $J=13.2$ and $\left.13.6 \mathrm{~Hz}, \mathrm{CH}_{2} \mathrm{Ph}\right), 3.79\left(\mathrm{~s}, 3 \mathrm{H}, \mathrm{OCH}_{3}\right), 3.92$ and $4.24\left(2 \mathrm{~d}, 2 \mathrm{H}, J=10\right.$ and $\left.9.6 \mathrm{~Hz}, \mathrm{CH}_{2} \mathrm{OH}\right), 5.36$ (br, $1 \mathrm{H}, \mathrm{NH}), 7.09\left(\mathrm{~m}, 2 \mathrm{H}, \mathbf{H}_{\mathrm{Ar}}\right), 7.29\left(\mathrm{~m}, 3 \mathrm{H}, \mathbf{H}_{\mathrm{Ar}}\right) ;{ }^{13} \mathrm{C} \mathrm{NMR}$ $\left(75 \mathrm{MHz}, \mathrm{CDCl}_{3}\right): 28.3\left[\left(\mathrm{CH}_{3}\right)_{3}\right], 37.8\left(\mathrm{CH}_{2} \mathrm{Ph}\right), 52.7$

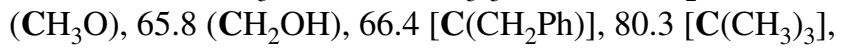
$127.2\left(\mathbf{C}_{\mathrm{HAr}}\right), 128.5\left(2 \mathbf{C}_{\mathrm{HAr}}\right), 129.8\left(2 \mathbf{C}_{\mathrm{HAr}}\right), 135.2\left(\mathbf{C}_{\mathrm{Ar}}\right)$, $155.1\left(\mathrm{NCO}_{2}\right), 172.4\left(\mathrm{CO}_{2} \mathrm{Me}\right)$. MS (ESI) $\mathrm{m} / z$ : $[\mathrm{M}+\mathrm{H}+]$ : 310.3 .

Synthesis of (RS)- $N$-(tertbutoxycarbonyl)-2,2-dimethyl-4-benzyl-4-methoxycarbonyl-3-oxazolidine (3)

A solution of 2 (15 g, $48.49 \mathrm{mmol})$, 2,2-dimethoxypropane $(10.09 \mathrm{~g}, 96.98 \mathrm{mmol})$ and $\mathrm{TsOH}(0.13 \mathrm{~g}, 0.67 \mathrm{mmol})$ in toluene $(177 \mathrm{~mL})$ was heated under reflux for $2 \mathrm{~h}$ and then slowly distilled during $15 \mathrm{~min}$ to eliminate the $\mathrm{MeOH}$ formed. DMP (10.09 g, $96.98 \mathrm{mmol}$ ) was then added and the procedure was repeated twice. After this time, the TLC showed no remaining starting material and clean formation of a single product. The cooled amber solution was partitioned between saturated sodium bicarbonate solution $(100 \mathrm{~mL})$ and ethyl ether $(100 \mathrm{~mL})$. The organic layer was washed with brine $(100 \mathrm{~mL})$, then dried with $\mathrm{Na}_{2} \mathrm{SO}_{4}$, filtered, and concentrated to give compound $\mathbf{3}$ as a yellow oil (15.6 g, $92 \%)$.

IR $(\mathrm{NaCl}): \quad 3,020 \quad\left(v_{\mathrm{CHAr}}\right), \quad 1,740 \quad\left(v_{\mathrm{C}=\text { Oester }}\right), \quad 1,698$ $\left(v_{\mathrm{C}=\text { Ocarbamate }}\right) ;{ }^{1} \mathrm{H}$ NMR $\left(300.13 \mathrm{MHz}, \mathrm{CDCl}_{3}\right): 0.74$, $0.91,1.48,1.53,1.55\left(5 \mathrm{~s}, 15 \mathrm{H}, 5 \mathrm{CH}_{3}\right), 3.06,3.1,3.14$, $3.76\left(4 \mathrm{~d}, 2 \mathrm{H}, J=10.5 \mathrm{~Hz}, \mathrm{CH}_{2} \mathrm{Ph}\right), 3.80,3.81(2 \mathrm{~s}, 3 \mathrm{H}$, $\left.\mathrm{OCH}_{3}\right), 4.05,4.07,4.09,4.11\left(4 \mathrm{~d}, 2 \mathrm{H}, \mathrm{CH}_{2} \mathrm{O}\right), 7.2(\mathrm{~d}, 2 \mathrm{H}$, $\left.J=7.2 \mathrm{~Hz}, \mathbf{H}_{\mathrm{Ar}}\right), 7.27\left(\mathrm{~m}, 3 \mathrm{H}, \mathbf{H}_{\mathrm{Ar}}\right) .{ }^{13} \mathrm{C} \mathrm{NMR}(75 \mathrm{MHz}$, $\left.\mathrm{CDCl}_{3}\right): 23.4,24.5,24.9,25.1,28.4\left(\mathrm{CH}_{3}\right), 37.2,39$ $\left(\mathrm{CH}_{2} \mathrm{Ph}\right), 52.6\left(\mathrm{CH}_{3} \mathrm{O}\right), 68.5,68.9\left[\mathrm{C}\left(\mathrm{CH}_{2} \mathrm{Ph}\right)\right], 70.3,71.2$ $\left(\mathbf{C H}_{2} \mathrm{O}\right), 80.5,80.7\left[\mathbf{C}\left(\mathrm{CH}_{3}\right)_{3}\right], 95.8,96.8\left[\mathbf{C}\left(\mathrm{CH}_{3}\right)_{2}\right], 126.8$, $126.9,127.3\left(\mathbf{C}_{\mathrm{HAr}}\right), 128,128.1,128.7\left(2 \mathbf{C}_{\mathrm{HAr}}\right), 129.8,131$, $131.1\left(2 \mathbf{C}_{\mathrm{HAr}}\right), 136.2,136.3\left(\mathbf{C}_{\mathrm{Ar}}\right), 150.8\left(\mathrm{NCO}_{2}\right), 172.2$ $\left(\mathrm{CO}_{2} \mathrm{Me}\right)$. MS (ESI) $m / z:[\mathrm{M}+\mathrm{H}+]: 350.3$.

Synthesis of (RS)- $N$-(tertbutoxycarbonyl)-2,2-dimethyl-4-benzyl-4-hydroxymethyl-3-oxazolidine (4)

Compound 3 (15.6 g, $44.65 \mathrm{mmol}$ ) was dissolved in $5 \%$ methanol in THF $(280 \mathrm{~mL})$ under a nitrogen atmosphere and cooled at $-12{ }^{\circ} \mathrm{C}$ with an ice-salt bath. Then lithium borohydride $(2.04 \mathrm{~g}, 93.74 \mathrm{mmol})$ is added by portions at such rate as to keep temperature below $-5^{\circ} \mathrm{C}$. The mixture was allowed to warm to room temperature with stirring overnight, after which TLC analysis indicates the reaction was complete. Quenching is performed by a slow addition of $200 \mathrm{~mL}$ of water at $0{ }^{\circ} \mathrm{C}$. The precipitate formed was filtered and washed with $40 \mathrm{~mL}$ of water. The filtrate was extracted three times with $60 \mathrm{~mL}$ of diethyl ether. The combined organic phases were washed with $50 \mathrm{~mL}$ of water, dried with $\mathrm{Na}_{2} \mathrm{SO}_{4}$, filtered, and evaporated under vacuum giving compound $\mathbf{4}$ as a yellow oil $(13.2 \mathrm{~g}, 92 \%)$.

IR (NaCl): 3,421 $\left(v_{\mathrm{OH}}\right), 1,691\left(v_{\mathrm{C}=\text { Ocarbamate }}\right) ;{ }^{1} \mathrm{H}$ NMR $\left(300.13 \mathrm{MHz}, \mathrm{CDCl}_{3}\right): 1.06,1.18,1.27,1.38,1.45(5 \mathrm{~s}$, $\left.15 \mathrm{H}, 5 \mathbf{C H}_{3}\right), 3.05,3.15\left(2 \mathrm{~d}, 2 \mathrm{H}, \mathrm{CH}_{2} \mathrm{Ar}, J=11.2 \mathrm{~Hz}\right)$, $3.44,3.90\left(2 \mathrm{~d}, 2 \mathrm{H}, \mathrm{CH}_{2} \mathrm{O}, J=9.3 \mathrm{~Hz}\right), 3.57$ and $3.70(2 \mathrm{~d}$, $\left.2 \mathrm{H}, J=11.7 \mathrm{~Hz}, \mathrm{CH}_{2} \mathrm{OH}\right), 4.75(\mathrm{br}, 1 \mathrm{H}, \mathrm{OH}), 7.20(\mathrm{~m}$,

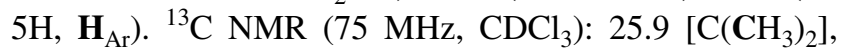
28.3 $\left[\left(\mathrm{CH}_{3}\right)_{3}\right], 37.8\left(\mathrm{CH}_{2} \mathrm{Ph}\right), 67.2\left(\mathrm{CH}_{2} \mathrm{OH}\right), 69.1\left(\mathrm{CH}_{2} \mathrm{O}\right)$, $81.1\left[\mathbf{C}\left(\mathrm{CH}_{3}\right)_{3}\right], 93.6\left[\mathbf{C}\left(\mathrm{CH}_{3}\right)_{2}\right], 95.6\left[\mathbf{C}\left(\mathrm{CH}_{2} \mathrm{Ph}\right)\right], 126.6$ $\left(\mathbf{C}_{\mathrm{HAr}}\right), 128.2\left(2 \mathbf{C}_{\mathrm{HAr}}\right), 130.5\left(2 \mathbf{C}_{\mathrm{HAr}}\right), 136.2\left(\mathbf{C}_{\mathrm{Ar}}\right), 154$ $\left(\mathrm{NCO}_{2}\right)$. MS (ESI) $\mathrm{m} / z$ : [M+H+]: 322.3 .

Synthesis of (RS)- $N$-(tertbutoxycarbonyl)-2,2dimethyl-4-benzyl-4-formyl-3-oxazolidine (5)

DMSO (7.58 mL, $106.78 \mathrm{mmol})$ was added to a solution of oxalyl chloride $(4.58 \mathrm{~mL}, 53.39 \mathrm{mmol})$ in $\mathrm{CH}_{2} \mathrm{Cl}_{2}$ $(130 \mathrm{~mL})$ at $-78{ }^{\circ} \mathrm{C}$. The resulting solution was stirred for $5 \mathrm{~min}$ at $-78{ }^{\circ} \mathrm{C}$ and then a solution of 4 (13.2 g, $41.07 \mathrm{mmol})$ in $\mathrm{CH}_{2} \mathrm{Cl}_{2}(45 \mathrm{~mL})$ was added. The resulting mixture was stirred for $15 \mathrm{~min}$ at $-78{ }^{\circ} \mathrm{C}$, and then $\mathrm{Et}_{3} \mathrm{~N}$ (33.63 mL, $242.31 \mathrm{mmol}$ ) was added. The solution was allowed to warm to room temperature overnight. The reaction was then quenched by addition of saturated $\mathrm{NaHCO}_{3}$ $(60 \mathrm{~mL})$ and then diluted with $\mathrm{Et}_{2} \mathrm{O}(70 \mathrm{~mL})$. The phases were separated, and the organic layer was washed with $1 \mathrm{M}$ 
of $\mathrm{KHSO}_{4}(100 \mathrm{~mL})$, saturated $\mathrm{NaHCO}_{3}(100 \mathrm{~mL})$, brine $(100 \mathrm{~mL})$, dried on sodium sulfate, filtered, and concentrated to give $\mathbf{5}$ which proved to be pure enough by TLC examination $(13.1 \mathrm{~g}, 92 \%)$.

IR (NaCl): 1,671 ( $\left.v_{\mathrm{C}=\text { Ocarbamate }}\right), 1,723\left(v_{\mathrm{C}=\text { Oaldehyde }}\right)$; ${ }^{1} \mathrm{H}$ NMR $\left(300.13 \mathrm{MHz}, \mathrm{CDCl}_{3}\right): 0.8,1.05,1.37,1.42$, $1.47\left(5 \mathrm{~s}, 15 \mathrm{H}, 5 \mathrm{CH}_{3}\right), 2.94,3.04,3.25$ and $3.5(4 \mathrm{~d}, 1 \mathrm{H}$, $\left.J=14 \mathrm{~Hz}, \mathrm{CH}_{2} \mathrm{Ph}\right), 3.85,3.90(2 \mathrm{~d}, 2 \mathrm{H}, J=14 \mathrm{~Hz}$, $\left.\mathrm{CH}_{2} \mathrm{O}\right), 7.2\left(\mathrm{~m}, 5 \mathrm{H}, \mathbf{H}_{\mathrm{Ar}}\right), 9.5(\mathrm{~s}, 1 \mathrm{H}, \mathrm{CHO}) ;{ }^{13} \mathrm{C} \mathrm{NMR}$ $\left(75 \mathrm{MHz}, \mathrm{CDCl}_{3}\right): 23.523 .6\left(\mathrm{CH}_{3}\right), 25.2\left(\mathrm{CH}_{3}\right), 27.327 .4$ $\left[\left(\mathrm{CH}_{3}\right)_{3}\right], 34.335 .8\left(\mathrm{CH}_{2} \mathrm{Ph}\right), 66.266 .3\left(\mathrm{CH}_{2} \mathrm{O}\right), 70.871 .2$ $\left[\mathbf{C}\left(\mathrm{CH}_{2} \mathrm{Ph}\right)\right], 80.280 .7\left[\mathbf{C}\left(\mathrm{CH}_{3}\right)_{3}\right], 94.495 .5\left[\mathbf{C}\left(\mathrm{CH}_{3}\right)_{2}\right]$, $125.8125 .9\left(\mathbf{C}_{\mathrm{HAr}}\right), 127.1127 .3\left(2 \mathbf{C}_{\mathrm{HAr}}\right), 129.8\left(2 \mathbf{C}_{\mathrm{HAr}}\right)$, $134.8134 .9\left(\mathbf{C}_{\mathrm{Ar}}\right), 149.8 \quad 151.2\left(\mathrm{NCO}_{2}\right), 197.1198 .7$ (CHO). MS (ESI) $m / z:[\mathrm{M}+\mathrm{Na}+]: 342.3$.

Synthesis of (RS)- $N$-(tertbutoxycarbonyl)-2,2-dimethyl-4-benzyl-4-ethynyl-3-oxazolidine (6)

To a solution of dimethyl (1-diazo-2-oxopropyl) phosphonate $(10.13 \mathrm{~g}, 57.54 \mathrm{mmol})$ and aldehyde 5 (12.25 g, $38.4 \mathrm{mmol})$ in dry methanol $(277 \mathrm{~mL})$ was added potassium carbonate $(10.6 \mathrm{~g}, 76.8 \mathrm{mmol})$ at $0{ }^{\circ} \mathrm{C}$ under nitrogen atmosphere for $1 \mathrm{~h}$. The mixture was allowed to warm to room temperature overnight, after which TLC analysis indicates the reaction was complete. After addition of aqueous saturated $\mathrm{NH}_{4} \mathrm{Cl}(900 \mathrm{~mL})$ and petroleum ether $(3 \times 900 \mathrm{~mL})$, the organic layer was separated, dried with $\mathrm{Na}_{2} \mathrm{SO}_{4}$, and evaporated to dryness. The crude product was purified by column chromatography (cyclohexane/ ethyl acetate: $95 / 5)$ to give $6(9.03 \mathrm{~g}, 74 \%)$ as an amber oil.

$R_{\mathrm{f}}$ (Cyclohexane/ethyl acetate: 95/5, v/v): 0.24; IR $(\mathrm{NaCl}): 3,289\left(v_{\text {CHalcyne }}\right), 1,694\left(v_{\mathrm{C}=\text { Ocarbamate }}\right) ;{ }^{1} \mathrm{H}$ NMR $\left(300.13 \mathrm{MHz}, \mathrm{CDCl}_{3}\right): 1.24,1.42,1.44,1.45,1.58[5 \mathrm{~s}$, $\left.15 \mathrm{H}, \mathrm{C}\left(\mathrm{CH}_{3}\right)_{3}\right], 2.41,2.45$ (2 s, 1H, Hacetylenic), 3.13 and 3.11, 3.34 (3d, $2 \mathrm{H}, J=13.2,13.6,13.2 \mathrm{~Hz}, \mathrm{CH}_{2} \mathrm{Ph}$ ), 3.95 and 4.09 ( $1 \mathrm{~d}$ and $\left.\mathrm{m}, 2 \mathrm{H}, J=9.2 \mathrm{~Hz} \mathrm{CH}_{2} \mathrm{O}\right), 7.28(\mathrm{~m}, 5 \mathrm{H}$,

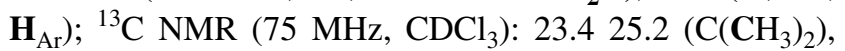
$28.1\left[\mathrm{C}\left(\mathrm{CH}_{3}\right)_{3}\right], 42.6\left(\mathrm{CH}_{2} \mathrm{Ph}\right), 59.459 .7\left[\mathbf{C}\left(\mathrm{CH}_{2} \mathrm{Ph}\right)\right]$, 71.6 (CHacetylenic), $71.8\left(\mathbf{C H}_{2} \mathrm{O}\right), 80.2\left[\mathbf{C}\left(\mathrm{CH}_{3}\right)_{3}\right], 83.6$ 83.7 (Cacetylenic), $9595.7\left[\mathbf{C}\left(\mathrm{CH}_{3}\right)_{2}\right], 126.5$ (CHAr), 127.6 (2CHAr), 130.3 (2CHAr), 135.5 (CAr), 150.8151 .0 $\left(\mathrm{NCO}_{2}\right)$. MS (ESI) $m / z:[\mathrm{M}+\mathrm{H}+]: 316.2$.

Synthesis of (RS)-2-amino-2-benzyl-but-3yn-1-ol hydrochloride (7)

A solution of $6(9 \mathrm{~g}, 28.53 \mathrm{mmol})$ and $6 \mathrm{~N} \mathrm{HCl}(145 \mathrm{~mL})$ was stirred at room temperature for $24 \mathrm{~h}$. After completion of the reaction (TLC examination), the solvent was then evaporated under reduced pressure to afford crude product which was dissolved and triturated in $\mathrm{Et}_{2} \mathrm{O}$. The organic layer was evaporated to afford 7 (5.61 g, $93 \%)$ as a white powder.

${ }^{1} \mathrm{H}$ NMR (400.13 MHz, $\left.\mathrm{D}_{2} \mathrm{O}\right): 3.18\left(\mathrm{~m}, 3 \mathrm{H}, \mathrm{CH}_{2} \mathrm{Ph}\right.$ and Hacetylenic), 3.77, $3.83\left(2 \mathrm{~d}, 2 \mathrm{H}, J=8\right.$ and $\left.7.6 \mathrm{~Hz} \mathrm{CH}_{2} \mathrm{O}\right)$, $7.43\left(\mathrm{~m}, 5 \mathrm{H}, \mathbf{H}_{\mathrm{Ar}}\right) ;{ }^{13} \mathrm{C}$ NMR $\left(100 \mathrm{MHz}, \mathrm{D}_{2} \mathrm{O}\right): 39.7$ $\left(\mathbf{C H}_{2} \mathrm{Ph}\right), 56.4\left[\mathbf{C}\left(\mathrm{CH}_{2} \mathrm{Ph}\right)\right], 64.4\left(\mathbf{C H}_{2} \mathrm{O}\right), 77.8(\mathbf{C H a c e t y}-$ lenic), 79.4 (Cacetylenic), 128.2 (CHAr), 128.8 (2CHAr), 130.9 (2CHAr), 132.9 (CAr). MS (ESI) $m / z:[\mathrm{M}+\mathrm{H}+]$ : 176.0 .

Synthesis of (RS)-2-[(tertbutoxycarbonyl)amino]-2benzyl-but-3-yn-1-ol (8)

$\mathrm{Et}_{3} \mathrm{~N}(6.38 \mathrm{~mL}, 47.84 \mathrm{mmol})$ and hydrochloride $7(2.53 \mathrm{~g}$, $11.96 \mathrm{mmol})$ were dissolved in dry THF $(20 \mathrm{~mL})$ and the mixture was stirred at $0{ }^{\circ} \mathrm{C}$ overnight. $\mathrm{Boc}_{2} \mathrm{O}(5.22 \mathrm{~g}$, $23.92 \mathrm{mmol})$ dissolved in THF $(10 \mathrm{~mL})$ was added dropwise to the mixture, and stirring was continued for $24 \mathrm{~h}$ at $0{ }^{\circ} \mathrm{C}$ and 3 days at $50{ }^{\circ} \mathrm{C}$. After completion of the reaction (TLC), the reaction mixture was filtered and washed with THF. After evaporation of THF, $\mathrm{Et}_{2} \mathrm{O}(100 \mathrm{~mL})$ and water $(50 \mathrm{~mL})$ were added. The organic phase was washed twice with $\mathrm{NaHCO}_{3}(80 \mathrm{~mL})$ and with brine $(80 \mathrm{~mL})$. The organic layer was separated, dried with $\mathrm{Na}_{2} \mathrm{SO}_{4}$, and evaporated to afford the crude product which was purified on column chromatography (cyclohexane/AcOEt: 8/2). The pure product 8 was isolated as a white powder $(1.10 \mathrm{~g}, 34 \%)$.

$R_{\mathrm{f}}$ (Cyclohexane/ethyl acetate: $\left.8 / 2, \mathrm{v} / \mathrm{v}\right): 0.33$; IR $(\mathrm{NaCl}): 3,275\left(v_{\text {CHalcyne }}\right), 3,236\left(v_{\mathrm{OH}}\right),\left(1,743\left(v_{\mathrm{C}=\text { Oester }}\right)\right.$, $1,680\left(v_{\mathrm{C}=\text { Ocarbamate }}\right) ;{ }^{1} \mathrm{H}$ NMR $\left(400.13 \mathrm{MHz}, \mathrm{CDCl}_{3}\right)$ : 1.39 (s, 9H, C(CH $\left.)_{3}\right), 2.37$ (s, 1H, Hacetylenic), 3.06, $3.21\left(2 \mathrm{~d}, 2 \mathrm{H}, J=9.2\right.$ and $\left.8.8 \mathrm{~Hz}, \mathrm{CH}_{2} \mathrm{Ph}\right), 3.71,3.82(2 \mathrm{~d}$, $\left.2 \mathrm{H}, J=7.2 \mathrm{~Hz} \mathrm{CH}_{2} \mathrm{O}\right), 4.83(\mathrm{~s}, 1 \mathrm{H}, \mathrm{NH}), 7.22(\mathrm{~m}, 5 \mathrm{H}$, $\left.\mathbf{H}_{\mathrm{Ar}}\right) ;{ }^{13} \mathrm{C} \mathrm{NMR}\left(100 \mathrm{MHz}, \mathrm{CDCl}_{3}\right): 28.5\left[\mathrm{C}\left(\mathrm{CH}_{3}\right)_{2}\right], 41$ $\left(\mathbf{C H}_{2} \mathrm{Ph}\right), 57.1\left[\mathbf{C}\left(\mathrm{CH}_{2} \mathrm{Ph}\right)\right], 68.1\left(\mathbf{C H}_{2} \mathrm{O}\right), 74.0$ (CHacetylenic), $80.6\left[\mathbf{C}\left(\mathrm{CH}_{3}\right)_{3}\right], 83.3$ (Cacetylenic), 127.2 (CHAr), 128.3 (2CHAr), 130.9 (2CHAr), 135.6 (CAr), 155.3 $\left(\mathrm{NCO}_{2}\right)$. MS (ESI) $m / z:[\mathrm{M}+\mathrm{H}+]: 276.1$.

Synthesis of (RS)-2-[(tertbutoxycarbonyl)amino]-2benzyl-but-3-ynoic acid (9)

Compound 8 (300 mg, $0.72 \mathrm{mmol}$ ) was dissolved in dichloromethane $(10 \mathrm{~mL})$ in a $25 \mathrm{~mL}$ flame-dried flask under nitrogen at $0{ }^{\circ} \mathrm{C}$. A solution of Dess-Martin periodinane in dichloromethane ( $15 \% \mathrm{wt}, 3 \mathrm{~mL}, 1.45 \mathrm{mmol}$ ) was added and the mixture was stirred for $2 \mathrm{~h}$ at $0{ }^{\circ} \mathrm{C}$. The reaction was filtered over a plug of silica and the plug was washed with a mixture ethyl acetate/cyclohexane 1:1. Concentration to dryness afforded the aldehyde which was used in the next step without further purification. The crude aldehyde was diluted in a mixture of tertbutanol/2-methyl-2-butene $(3: 1,20 \mathrm{~mL})$ and the resulting solution was cooled to 
$0{ }^{\circ} \mathrm{C}$. A solution of sodium chlorite $(417 \mathrm{mg}, 4.6 \mathrm{mmol})$ and sodium phosphate monobasic $(410 \mathrm{mg}, 2.63 \mathrm{mmol})$ in water $(5 \mathrm{~mL})$ was slowly added, and the reaction was stirred for $30 \mathrm{~min}$ at room temperature. The mixture was diluted with water and extracted with ethylacetate. The combined organic layers were dried with $\mathrm{Na}_{2} \mathrm{SO}_{4}$, filtered, and concentrated to dryness. The crude product was purified by column chromatography (dichloromethane/Methanol: $8 / 2)$ to afford of $9(100 \mathrm{mg}, 32 \%)$ as a colorless oil.

$R_{\mathrm{f}}$ (dichloromethane/methanol: $\left.8 / 2, \quad \mathrm{v} / \mathrm{v}\right): 0.58 ;$ IR $(\mathrm{NaCl}): 3,403\left(v_{C H \text { alcyne }}\right), 3,289\left(v_{O H}\right), 1,610\left(v_{C=O \text { acid }}\right)$, $\left(1,692\left(v_{C=O \text { carbamate }}\right) ;{ }^{1} \mathrm{H}\right.$ NMR $\left(400.13 \mathrm{MHz}\right.$, Acetone-D $\left.{ }^{6}\right)$ : 1.31 (s, 9H, C( $\left.\left.\mathrm{CH}_{3}\right)_{3}\right), 2.51$ (s, 1H, Hacetylenic), 3.45 (m, $\left.2 \mathrm{H}, \mathrm{CH}_{2} \mathrm{Ph}\right), 7.06\left(\mathrm{~m}, 5 \mathrm{H}, \mathbf{H}_{\mathrm{Ar}}\right) ;{ }^{13} \mathrm{C}$ NMR $(100 \mathrm{MHz}$, Acetone-D $\left.{ }^{6}\right): \quad 28.8 \quad\left[\mathrm{C}_{\left.\left(\mathbf{C H}_{3}\right)_{2}\right],} \quad 42.15 \quad\left(\mathbf{C H}_{2} \mathrm{Ph}\right), \quad 58.5\right.$ $\left[\mathbf{C}\left(\mathrm{CH}_{2} \mathrm{Ph}\right)\right], 71.3$ (CHacetylenic), $78.7\left[\mathrm{C}\left(\mathrm{CH}_{3}\right)_{3}\right], 84.8$ (Cacetylenic), 126.4 (CHAr), 127.9 (2CHAr), 130.8 (2CHAr), 136.8 (CAr), $153.9\left(\mathrm{NCO}_{2}\right), 173.4\left(\mathbf{C O}_{2} \mathrm{H}\right)$. MS (ESI) $m / z:[\mathrm{M}+\mathrm{H}+]: 290$.

Synthesis of (RS)-methyl 2-[(tertbutoxycarbonyl) amino]-2-benzyl-but-3-ynoate (10)

The acid 9 ( $80 \mathrm{mg}, 0.28 \mathrm{mmol})$ was dissolved in THF $(5 \mathrm{~mL})$ and Methanol $(2.5 \mathrm{~mL})$ and cooled at $0{ }^{\circ} \mathrm{C}$. Trimethylsilyldiazomethane $(2.0 \mathrm{M}$ in hexane, $0.29 \mathrm{~mL}$, $0.56 \mathrm{mmol}$ ) was added dropwise over $10 \mathrm{~min}$ and the solution was slowly warmed to room temperature overnight. The mixture was concentrated in vacuo and the crude product was taken up in ethyl acetate, washed with $\mathrm{NaHCO}_{3}$, water, and brine, dried with $\mathrm{Na}_{2} \mathrm{SO}_{4}$ and concentrated to dryness. The crude product was purified by column chromatography (Ethyl acetate/Petroleum ether: 5/95) to give compound $\mathbf{1 0}$ as a clear oil which solidified upon standing (21 mg, $25 \%$ ).

$\mathrm{R}_{\mathrm{f}}$ (Cyclohexane/ethyl acetate: 8/2, v/v): 0.64; IR ( $\left.\mathrm{NaCl}\right)$ : $3,379\left(v_{\text {CHalcyne }}\right), 1,743\left(v_{\mathrm{C}=\text { Oester }}\right), 1,710\left(v_{\mathrm{C}=\text { Ocarbamate }}\right) ;{ }^{1} \mathrm{H}$ NMR (300.13 MHz, Acetone-D $\left.)^{6}\right): 1.47$ [s, 9H, C( $\left.\left(\mathbf{C H}_{3}\right)_{3}\right]$, 3.04 (s, 1H, Hacetylenic), 3.40, $3.47(2 \mathrm{~d}, 2 \mathrm{H}, J=12 \mathrm{~Hz}$ $\left.\mathrm{CH}_{2} \mathrm{Ph}\right), 3.75\left(\mathrm{~s}, 3 \mathrm{H}, \mathrm{OCH}_{3}\right), 6.41(\mathrm{~s}, 1 \mathrm{H}, \mathrm{NH}), 7.31(\mathrm{~m}$, $\left.5 \mathrm{H}, \mathbf{H}_{\mathrm{Ar}}\right) ;{ }^{13} \mathrm{C}$ NMR $\left(75 \mathrm{MHz}\right.$, Acetone-D $\left.{ }^{6}\right): 28.5\left[\mathrm{C}\left(\mathrm{CH}_{3}\right)_{2}\right]$, $43.3\left(\mathbf{C H}_{2} \mathrm{Ph}\right), 53.2\left(\mathrm{OCH}_{3}\right), 59.1\left[\mathbf{C}\left(\mathrm{CH}_{2} \mathrm{Ph}\right)\right], 75.1(\mathbf{C H a}-$ cetylenic), $80.1\left[\mathbf{C}\left(\mathrm{CH}_{3}\right)_{3}\right], 82.1$ (Cacetylenic), 128 (CHAr),
128.8 (2CHAr), 131.4 (2CHAr), 135.9 (CAr), $154.8\left(\mathrm{NCO}_{2}\right)$, $170.4\left(\mathrm{CO}_{2} \mathrm{CH}_{3}\right)$. MS (ESI) $m / z$ : [M+Na]: 326 . HRMS calcd. for $\mathrm{C}_{17} \mathrm{H}_{21} \mathrm{NO}_{4} \mathrm{Na}$ : 326.1371, found 326.1368.

Infrared (IR) and vibrational circular dichroism (VCD) spectra were recorded on a Bruker PMA 50 accessory coupled to a Vertex70 Fourier transform infrared spectrometer. A photoelastic modulator (Hinds PEM 90) set at 1/4 retardation was used to modulate the handedness of the circular polarized light at $50 \mathrm{kHz}$. Demodulation was performed by a lock-in amplifier (SR830 DSP). An optical low-pass filter $\left(<1,800 \mathrm{~cm}^{-1}\right)$ before the photoelastic modulator was used to enhance the signal/noise ratio. All solutions were prepared from $5.6 \mathrm{mg}$ in $185 \mathrm{~mL} \mathrm{CD} \mathrm{Cl}_{2}\left(\mathrm{C}=0.1 \mathrm{~mol} . \mathrm{L}^{-1}\right)$. For the individual spectra of the enantiomers, about 12,000 scans were averaged at $4 \mathrm{~cm}^{-1}$ resolution (corresponding to three hours of measurement time). The baselines of the VCD spectra were corrected using the half subtraction of the raw spectra of the two enantiomers. For the infrared spectra, the cell filled with $\mathrm{CD}_{2} \mathrm{Cl}_{2}$ served as reference. The spectra are presented without smoothing and further data processing.

All calculations were performed using the $R$ enantiomer of the molecule 10. The optimized geometries, vibrational frequencies, IR absorption, and VCD intensities were calculated using Density Functional Theory (DFT) with SMD-B3PW91/6-311 + G(df, p). The population of each conformation was estimated using Boltzmann's statistics. Frequencies were scaled by a factor of 0.98 . IR absorption and VCD spectra were constructed from calculated dipole and rotational strengths assuming a Lorentzian band shape with a half-width at half maximum of $12 \mathrm{~cm}^{-1}$.

The HPLC screening of chiral stationary phases was achieved on a unit composed of a Merck D-7000 system manager, a Merck-Lachrom L-7100 pump, a MerckLachrom L-7200 autosampler, a Merck-Lachrom L-7360 oven, a Merck-Lachrom L-7400 UV-detector.

Semipreparative separation of $\mathbf{1 0}$ was achieved on LuxCellulose-2 $(250 \times 4.6 \mathrm{~mm}, 5 \mathrm{~mm})$, a Cellulose tris(3chloro-4-methylphenylcarbamate) coated on silica available from Phenomenex, on an unit composed of a Merck D-7000 system manager, a Merck-Hitachi L-6000 pump, a Rheodyne valve with a $2 \mathrm{~mL}$ loop and a Merck-Hitachi L-4000 UV-detector.
Scheme 1 Synthesis of DL$N$-Boc- $N, O$-isopropylidene- $\alpha$ benzylserinal (5)

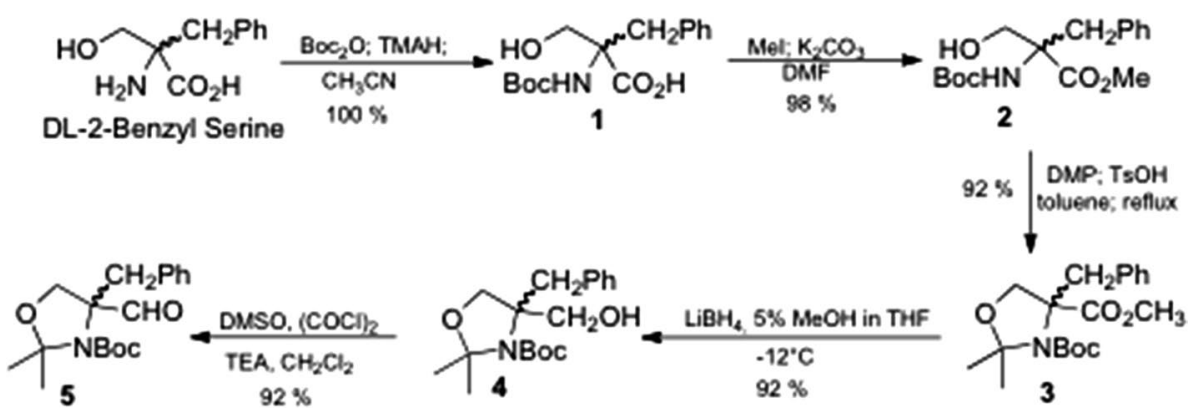


Scheme 2 Synthesis of DL- $N$-(tertbutoxycarbonyl)- $\alpha$ ethynylphenylalanine methyl ester (10)
Table 1 Screening results for the separation of the enantiomers of $\mathbf{1 0}$ on 16 different chiral stationary phases

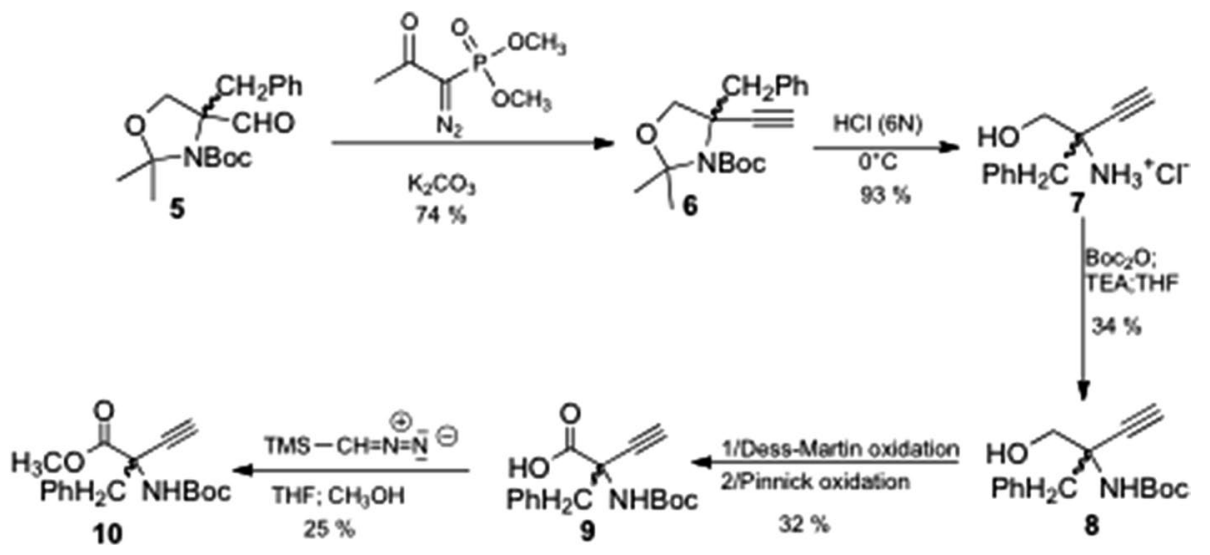

\begin{tabular}{|c|c|c|c|c|c|c|c|}
\hline Column & Mobile phase & $\mathrm{t} R$ & $\mathrm{k} R$ & $\mathrm{t} S$ & $\mathrm{k} S$ & $\alpha$ & Rs \\
\hline \multirow[t]{2}{*}{ Chiralpak AS-H } & Heptane/ethanol 90/10 & 4.58 & 0.53 & - & - & 1 & 0 \\
\hline & Heptane/isopropanol 90-10 & 5.22 & 0.74 & - & - & 1 & 0 \\
\hline \multirow[t]{2}{*}{ Chiralcel OD-3 } & Heptane/ethanol 90-10 & 5.29 & 0.76 & 5.46 & 0.82 & 1.08 & 0.51 \\
\hline & Heptane/isopropanol 90/10 & 6.96 & 1.32 & - & - & 1 & 0 \\
\hline \multirow[t]{2}{*}{ Chiralcel OJ-H } & Heptane/ethanol 90/10 & 8.15 & 1.72 & 6.76 & 1.25 & 1.38 & 2.56 \\
\hline & Heptane/isopropanol 90/10 & 7.95 & 1.65 & 6.87 & 1.29 & 1.28 & 1.27 \\
\hline \multirow[t]{2}{*}{$(S, S)$-Whelk-O1 } & Heptane/ethanol 90/10 & 7.06 & 1.35 & - & - & 1 & 0 \\
\hline & Heptane/isopropanol 90/10 & 7.96 & 1.65 & - & - & 1 & 0 \\
\hline \multirow[t]{2}{*}{$(S, S)$-Ulmo } & Heptane/ethanol 90/10 & 4.72 & 0.57 & - & - & 1 & 0 \\
\hline & Heptane/isopropanol 90/10 & 4.75 & 0.58 & - & - & 1 & 0 \\
\hline \multirow[t]{2}{*}{ Chiralpak AZ-H } & Heptane/ethanol 90/10 & 6.97 & 1.32 & 7.35 & 1.45 & 1.10 & 1.11 \\
\hline & Heptane/isopropanol 90/10 & 8.22 & 1.74 & 8.68 & 1.89 & 1.09 & 1.05 \\
\hline \multirow[t]{2}{*}{ Lux-Cellulose-2 } & Heptane/ethanol 90/10 & 6.21 & 1.07 & 7.33 & 1.44 & 1.35 & 4.26 \\
\hline & Heptane/isopropanol 90/10 & 7.62 & 1.54 & 8.88 & 1.96 & 1.27 & 3.41 \\
\hline \multirow[t]{2}{*}{ Chiralpak AD-H } & Heptane/ethanol 90/10 & 7.43 & 1.48 & - & - & 1 & 0 \\
\hline & Heptane/isopropanol 90/10 & 9.49 & 2.16 & 10.11 & 2.37 & 1.10 & 1.21 \\
\hline \multirow[t]{2}{*}{ Lux-Amylose-2 } & Heptane/ethanol 90/10 & 8.03 & 1.68 & 9.86 & 2.29 & 1.36 & 4.44 \\
\hline & Heptane/isopropanol 90/10 & 11.76 & 2.92 & 14.83 & 3.94 & 1.35 & 4.39 \\
\hline \multirow[t]{2}{*}{ Lux-Cellulose-4 } & Heptane/ethanol 90/10 & 5.92 & 0.97 & 7.10 & 1.37 & 1.41 & 4.16 \\
\hline & Heptane/isopropanol 90/10 & 7.68 & 1.56 & 9.43 & 2.14 & 1.37 & 4.19 \\
\hline \multirow[t]{2}{*}{ Chiralpak IA } & Heptane/ethanol 90/10 & 5.45 & 0.82 & 5.71 & 0.90 & 1.10 & 0.92 \\
\hline & Heptane/isopropanol 90/10 & 6.24 & 1.08 & 6.63 & 1.21 & 1.12 & 1.19 \\
\hline \multirow[t]{2}{*}{ Chiralpak IB } & Heptane/ethanol 90/10 & 4.94 & 0.65 & - & - & 1 & 0 \\
\hline & Heptane/isopropanol 90/10 & 5.41 & 0.80 & - & - & 1 & 0 \\
\hline \multirow[t]{2}{*}{ Chiralpak IC } & Heptane/ethanol 90/10 & 6.20 & 1.07 & 5.96 & 0.99 & 1.08 & \\
\hline & Heptane/isopropanol 90/10 & 10.51 & 2.50 & 8.71 & 1.90 & 1.32 & 2.58 \\
\hline \multirow[t]{2}{*}{ Chiralpak ID } & Heptane/ethanol 90/10 & 6.66 & 1.22 & - & - & 1 & 0 \\
\hline & Heptane/isopropanol 90/10 & 9.42 & 2.14 & - & - & 1 & 0 \\
\hline \multirow[t]{2}{*}{ Chiralpak IE } & Heptane/ethanol 90/10 & 7.37 & 1.46 & - & - & 1 & 0 \\
\hline & Heptane/isopropanol 90/10 & 8.30 & 1.77 & 8.61 & 1.87 & 1.06 & 0.72 \\
\hline \multirow[t]{2}{*}{ Chiralpak IF } & Heptane/ethanol 90/10 & 6.69 & 1.23 & 6.93 & 1.31 & 1.07 & 0.46 \\
\hline & Heptane/isopropanol 90/10 & 7.05 & 1.35 & 7.37 & 1.46 & 1.08 & 0.81 \\
\hline
\end{tabular}


- Sample preparation: about $13 \mathrm{mg}$ of compound $\mathbf{1 0}$ are dissolved in $2 \mathrm{~mL}$ of ethanol.

- Chromatographic conditions: Lux-Cellulose-2 $(250 \times 10 \mathrm{~mm})$, hexane/2-PrOH (9/1) as mobile phase, flow-rate $=5 \mathrm{~mL} / \mathrm{min}$, UV detection at $210 \mathrm{~nm}$.

- Injections: 10 times $200 \mathrm{~mL}$, every $10 \mathrm{~min}$.

- Collection: the first eluted enantiomer is collected between 7 and 8 min and the second one between 8 and $10 \mathrm{~min}$.

- First fraction: $5.6 \mathrm{mg}$ of the first eluted enantiomer, $\alpha_{\mathrm{D}}^{25}=+12\left(c=0.56, \mathrm{CHCl}_{3}\right)$ with ee $>99.5 \%$

- Second fraction: $5.9 \mathrm{mg}$ of the second eluted enantiomer, $\alpha_{\mathrm{D}}^{25}=-12\left(c=0.59, \mathrm{CHCl}_{3}\right)$ with ee $=98.8 \%$.

Heptane, 2-PrOH and EtOH, HPLC grade, were degassed and filtered on a $0.45 \mathrm{~mm}$ Millipore membrane before use. Retention times Rt in minutes, retention factors $k_{i}=\left(\mathrm{Rt}_{i}-\mathrm{Rt}_{0}\right) / \mathrm{Rt}_{0}$, enantioselectivity factor $a=k_{2} / k_{1}$, and resolution $\mathrm{Rs}=2\left(\mathrm{Rt}_{2}-\mathrm{Rt}_{1}\right) /\left(w_{1}+w_{2}\right)$ are given in the Table of data. $\mathrm{Rt}_{0}$ was determined by injection of tri-tertiobutyl benzene and wi was the width of the peak.

\section{Results and discussion}

(RS)- $N$-(tertbutoxycarbonyl)-4-formyl-2,2-dimethyl-4-benzyl-3-oxazolidine (5) was prepared from DL-2-benzylserine in five steps, in good overall yield (76\%), following a modification of the method developed by us (Meffre et al. 1994) (Scheme 1). Treatment of the commercially available DL2-benzylserine with ditertbutyldicarbonate under standard conditions (sodium hydroxyde as base in a mixture of dioxane/water as solvent) did not afford the expected $N$-Boc protected amino acid $\mathbf{1}$. For this reason, we used the lipophilic base tetramethylammonium hydroxide to solubilize the zwitterionic amino acid in acetonitrile (Khalil et al. 1996). Using this procedure DL-2-[(tertbutoxycarbonyl)amino]-2-benzylserine 1 was obtained in quantitative yield. Esterification of 1 with methyl iodide and solid potassium carbonate in DMF afforded compound $\mathbf{2}$ in $98 \%$ yield without any further purification. Compound 2 was converted into oxazolidine $\mathbf{3}$, in $92 \%$ yield, using 2,2-dimethoxypropane (DMP) in toluene at reflux with p-toluenesulfonic acid $(\mathrm{TsOH})$ as catalyst (Garner and Park 1992). Reduction of $\mathbf{3}$ was performed with lithium borohydride in a mixture of THF/MeOH $(95 / 5$, $\mathrm{V} / \mathrm{V}$ ) at $-12{ }^{\circ} \mathrm{C}$ to give alcohol 4 in $92 \%$ yield after column chromatography purification. Compound $\mathbf{4}$ was finally oxidized under Swern conditions (Meffre et al. 1994; Outten and Daves 1989) to obtain the required aldehyde $\mathbf{5}$ in good yield $(92 \%)$. Building block 5 was prepared on more than ten gram scale and used after work up without any purification. These compounds were characterized by ${ }^{1} \mathrm{H}$ NMR, ${ }^{13} \mathrm{C}$ NMR, and by low resolution mass spectroscopies.
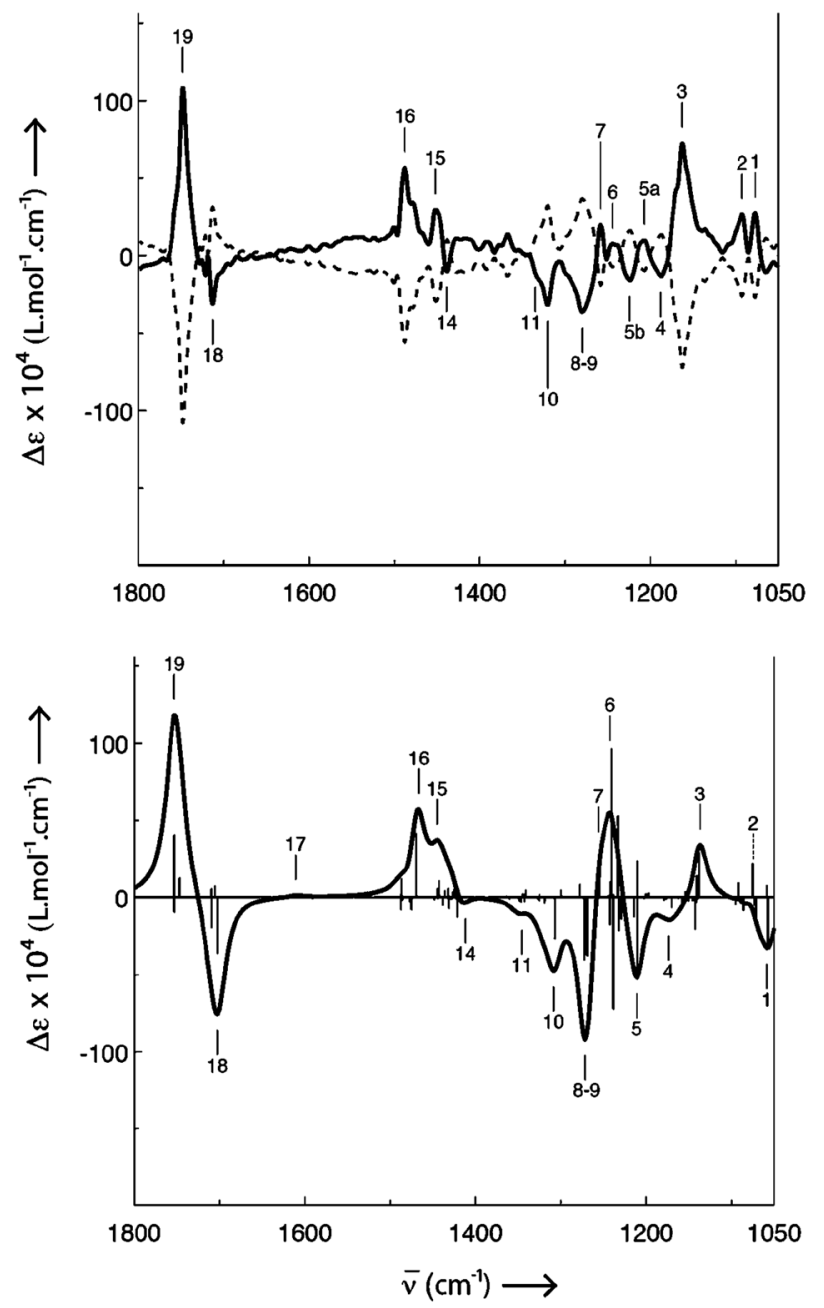

Fig. 1 (Top) experimental VCD spectra for the first eluted enantiomer of $\mathbf{1 0}$ on Lux-Cellulose-2 (green) and for the second eluted enantiomer of $\mathbf{1 0}$ on Lux-Cellulose-2 (dotted red). (Bottom) calculated VCD spectrum for $(R)$-enantiomer of $\mathbf{1 0}$ (color figure online)

We use Bestmann-Ohira method to obtain alkyne 6 in $74 \%$ yield after purification by column chromatography because of the mild conditions applied (Ohira 1989; Muller et al. 1996, Meffre et al. 2002) (Scheme 2). Several attempts to carry out the selective deprotection of the isopropylidene group of 6 failed: $\mathrm{Sc}(\mathrm{OTf})_{3}(10 \mathrm{~mol} \%)$ (Avenoza et al. 1999b), $\mathrm{CuCl}_{2} \cdot 2 \mathrm{H}_{2} \mathrm{O}$ (Venkataiah et al. 2011), $\mathrm{BiBr}_{3}$ (Cong et al. 2005), Pyridinium paratoluenesulfonate (Clerc et al. 2010). The successfulness of this transformation is known to be highly substrate dependant. We were pleased to observe that both deprotection of the N-Boc and isopropylidene groups using $6 \mathrm{~N}$ hydrochloride acid $\left(24 \mathrm{~h}\right.$ at $\left.0{ }^{\circ} \mathrm{C}\right)$ proceeded in almost quantitative yield to give the amino alcohol hydrochloride 7. Different methods of amine protection (sodium hydroxyde as base in a mixture of dioxane/water as solvent, TMAH in acetonitrile) 
were tried without any success. Amino protection proved to be difficult. Best results were observed when compound 7 was then protected with $\mathrm{Boc}_{2} \mathrm{O}$ in the presence of TEA in THF to give $\mathbf{8}$ in $34 \%$ after purification by column chromatography.

Final oxidation step is also known to be highly substrate dependent. A two step oxidation of alcohol 8 was performed to obtain acid 9. In the first step, Dess-Martin periodinane was used to provide the intermediate aldehyde (not isolated), which was then immediately oxidized to the corresponding acid derivative 9 by a Pinnick oxidation procedure (Bal et al. 1981; Lane and Halcomb 2003). The racemic resolution of 9 by chiral chromatography was tempted but decomposition of 9 was observed in the chiral chromatography conditions (TFA). For this reason, the quaternary amino acid 9 was esterified by $\mathrm{TMSCHN}_{2}$ (Shiau et al. 2009) to give 10. After screening of 16 different chiral stationary phases (Table 1), enantiomers of $\mathbf{1 0}$ were successfully separated under analytical conditions, on a LuxCellulose-2 column and under semipreparative conditions. The absolute configuration of the first eluted enantiomer was assigned by comparison of the experimental VCD spectrum with the calculated spectrum for $(R)-\mathbf{1 0}$ (Fig. 1).

\section{Conclusion}

In summary, we have developed a new and efficient method for the synthesis of racemic-protected $\alpha$-ethynylphenylalanine starting from DL-2-benzylserine, in ten steps. Resolution of the racemic was performed by HPLC techniques using a chiral stationary phase and specific optical rotations were measured. The absolute configurations of the separated enantiomers were determined by VCD. $\alpha$-ethynylphenylalanine represents an interesting, chiral building block for its incorporation in peptides. Further research will study its biological properties.

Acknowledgments We gratefully thank the French "ministère de l'éducation nationale, de l'enseignement supérieur et de la recherche" for its financial support. We thank graduate students for their help in the synthesis: Magali Blum, Clément Bonnel.

Conflict of interest The authors declare that they have no conflict of interest.

\section{References}

Almond HR, Manning DT, Niemann C (1962) Interaction of alphachymotrypsin with several alpha-methyl alpha-acylamino acid methyl esters. Biochemistry 1:243

Avenoza A, Cativiela C, Peregrina J, Sucunza D, Zurbano M (1999a) A straightforward synthesis of both enantiomers of $\alpha$-vinylalanine and $\alpha$-ethynylalanine. Tetrahedron Asymmetry 10:4653-4661
Avenoza A, Cativiela C, Corzana F, Peregrina J, Zurbano M (1999b) Preparation and synthetic applications of (S)- and (R)- $N$-Boc$N, O$-isopropylidene- $\alpha$-methylserinals: asymmetric synthesis of (S)- and (R)-2-Amino-2-methylbutanoic acids (Iva). J Org Chem 64:8220-8225

Avenoza A, Cativiela C, Corzana F, Peregrina J, Sucunza D, Zurbano M (2001) Enantioselective synthesis of (S)-and (R)- $\alpha$ methylserines:application to the synthesis of (S)- and (R)- $N$-Boc$N, O$-isopropylidene- $\alpha$-methylserinals. Tetrahedron Asymmetry 12:949-957

Bal BS, Childers W, Pinnick X (1981) Oxydation of $\alpha, \beta$-unsaturated aldehydes. Tetrahedron 37:2091-2096

Berkowitz DB, Chisowa E, McFadden JM (2001) Stereocontrolled synthesis of quaternary $\beta, \gamma$-unsaturated amino acids: chain extension of $d$ - and 1- $\alpha$-(2-tributylstannyl)vinyl amino acids. Tetrahedron 57:6329

Berkowitz DB, De la Salud-Bea R, Jahng W (2004) Synthesis of quaternary Amino acids bearing a (2'Z)-fluorovinyl $\alpha$-branch:potential PLP enzymes inactivators. Org Lett 6(11):1821-1824

Bouifraden S, Drouot C, El Hadrami M, Guenoun F, Lecouinte L, Mai N, Paris M, Pothion C, Sadoune M, Sauvagnat B, Amblard M, Aubagnac JL, Calmes M, Chevallet P, Daunis J, Enjalbal C, Fehrentz JA, Lamaty F, Lavergne JP, Lazaro R, Rolland V, Roumestand ML, Viallefont P, Vidal Y, Martinez J (1999) Some of the amino acid chemistry going on in laboratory of amino acids, peptides and proteins. Amino Acids 16:345

Casara P, Metcalf BW (1978) Trimethyl acetylene- $N$-carboethoxy glycinate dianion. a general synthon for $\alpha$-acetylenic $\alpha$-amino acids. Tetrahedron Lett 18:1581-1584

Cativiela C, Diaz de Villegas MD (2000) Stereoselective synthesis of quaternary $\alpha$;-amino acids. Part 2 : cyclic compounds. Tetrahedron Asymmetry 11:645-732

Cativiela C, Diaz de Villegas MD (2007) Recent progress on the stereoselective synthesis of acyclic quaternary $\alpha$-amino acids. Tetrahedron Asymmetry 18:569-623

Clerc J, Schellenberg B, Groll M, Bachmann A, Huber R, Dudler R, Kaiser M (2010) Convergent synthesis and biological evaluation of syringolin A and derivatives as eukaryotic $20 \mathrm{~S}$ proteasome inhibitors. Eur J Org Chem 21:3991-4003

Colson PJ, Hegedus LS (1993) Asymmetric synthesis of $\alpha$-alkyl-aamino acids from chromium-carbene-complex-derived -lactams. J Org Chem 58:5981

Cong X, Hu F, Liu K-G, Liao Q, Yao Z (2005) Chemoselective deprotection of cyclic $N, O$-aminals using catalytic bismuth(III) bromide in acetonitrile. J Org Chem 70:4514-4516

Crucianelly M, De Angelis F, Lazzaro F, Malpezzi L, Volonterio A, Zanda M (2004) Synthesis of enantiomerically pure $\alpha$-ethyl, $\alpha$-vinyl and $\alpha$-ethynyl 3,3,3-trifluoro alaninates. J Fluorine Chem 125:573-577

Danzin C, Casara P, Claverie N, Metcalf BW (1981) $\alpha$-ethynyl and $\alpha$-vinyl analogues of ornithine as enzyme-activated inhibitors of mammalian ornithine decarboxylase. J Med Chem 24:16

Di Giacomo M, Vinci V, Serra M, Colombo L (2008) New fast and practical method for the enantioselective synthesis of $\alpha$-vinyl, $\alpha$-alkyl quaternary $\alpha$-amino acids. Tetrahedron Asymmetry 19:247-257

Duggan HME, Hitchcock PB, Young DW (2005) Synthesis of 5/7-, 5/8- and 5/9-bicyclic lactam templates as constraints for external $\alpha$-turns. Org Biomol Chem 3(12):2287

Finkbeiner P, Weckenmann NM, Nachtsheim BJ (2014) Alkynyliodonium salt mediated alkynylation of azlactones: fast access to $c \alpha$-tetrasubstituted $\alpha$-amino acid derivatives. Org Lett 16:1326-1329

Garner P, Park JM (1992) 1,1,-Dimethylethyl(S) or (R)-4-formyl2,2-dimethyl-3-oxazolinecarboxylate:a usefullserinal derivative. Org Synth 70:18-25 
Gonzalez DF, Brand JP, Waser J (2010) Ethynyl-1,2-benziodoxol$3(1 \mathrm{H})$-one $(\mathrm{EBX})$ : an exceptional reagent for the ethynylation of keto, cyano, and nitro esters. Chem Eur J 16:9457-9461

Hruby VJ, Li G, Haskell-Luevano C, Shenderovich MD (1997) Design of peptides proteins, and peptidomimetics in Chi space. Biopolymers Peptide Sci. 43:219-266

Johansson H, Pedersen D (2012) Azide- and alkyne-derivatised $\alpha$-amino acids. Eur J Org Chem 2012:4267-4281

Kaiser J, Kinderman SS, Van Esseveldt BC, Van Delft FL, Schoemaker HE, Blaauw RH, Rutjes FPJT (2005) Synthetic applications of aliphatic unsaturated $\alpha-\mathrm{H}-\alpha$-amino acids. Org Biomol Chem 3:3435

Keith DD, Tortora JA, Ineiken KS, Leimbgruber W (1975) The total synthesis of rhizobitoxine. Tetrahedron 31:2633

Khalil EM, Subasinghe NL, Johnson RL (1996) An efficient and high yield method for the $N$-tert-butoxycarbonyl protection of sterically hindered amino acids. Tetrahedron Lett 37(20):3441-3444

Khosla MC, Stachowiak K, Smeby RR, Bumpus FM, Piriou F, Lintner K, Fermandjian S (1981) Synthesis of [a-methyltyrosine-4] angiotensin II: studies of its conformation, pressor activity, and mode of enzymatic degradation. Proc Natl Acad Sci USA Biol 78:757

Kuroda Y, Okuhara M, Goto T, Iguchi E, Kohsaka M, Aoki H, Imanaka H (1980) FR-900148, a new antibiotic. I. Taxonomy, fermentation, isolation and characterization. J Antibiot:125-131

Kuroda Y, Okuhara M, Goto T, Iguchi E, Kohsaka M, Aoki H, Imanaka H (1980b) FR-900130, a novel amino acid antibiotic. II. Isolation and structure elucidation of the acetyl derivative of FR-900130. J Antibiot 33:132

Lane J, Halcomb RA (2003) New method for the stereoselective synthesis of $\alpha$-substituted serine amino acid analogues. Org Lett 5(22):4017-4020

Maycock AL, Aster SD, Patchett A (1979) A Suicide inactivation of decarboxylases. Dev Biochem 6:115

Meffre P, Legoffic F (1996) $\beta, \gamma$-Alkynyl $\alpha$-amino acids: a synthetic challenge. Amino Acids 11:313-328

Meffre P, Durand P, Branquet E, Legoffic F (1994) A straightforward synthesis of $N$-Boc-L-serinal and $N$-Boc-L-threoninal acetonides. Synth Commun 24(15):2147-2152

Meffre P, Hermann S, Durand P, Reginato G, Riu A (2002) Practical one-step synthesis of ethynylglycine synthon from Garner's aldehyde. Tetrahedron 58:5159-5162

Metcalf BW, Jund K (1977) Synthesis of $\beta, \gamma$-unsaturated amino acids as potential catalytic irreversible enzyme inhibitors. Tetrahedron Lett 18(41):3689

Muller S, Liepold B, Roth GJ, Bestmann HJ (1996) An improved One-pot procedure for the synthesis of alkynes from aldehydes. Synlett 1996:521-522

O'Connor SJ, Liu Z (2003) A concise synthesis of sterically hindered 3-amino-2-oxindoles. Synlett 2003:2135

Ohira S (1989) Methanolysis of dimethyl (1-diazo-2oxopropyl) phosphonate:generation of dimethyl (diazomethyl) phosphonate and reaction with carbonyl compounds. Synth Commun 19:561

Olma A, Lasota A, Kudaj A (2012) A convenient synthesis to optically pure $\alpha$-alkyl- $\beta$-(sec-amino)alanines. Amino Acids 42:2525-2528
Ortial S, Dave R, Benfodda Z, Bénimélis D, Meffre P (2014) Synthesis of cis-5-trifluoromethylproline from L-glutamic acid. Synlett 25:569-573

Outten RA, Daves GD (1989) Benzo[d]naphtho[1,2-b]pyran-6-one C-Glycosides. Aryltri- $n$-butylstannanes in palladium-mediated coupling with 2,3-dihydropyran and furanoid glycals. J Org Chem 54:29

Patchett AA, Taub D (1980) alpha-ethynyle-alpha-amino-acides et leurs esters et compositions pharmaceutiques les contenant, EP0008658A1

Polinelli S, Broxterman QB, Schoemaker HE, Boesten WHJ, Crisma M, Valle G, Toniolo C, Kamphuis J (1992) New aspartame-like sweeteners containing L- $(\alpha \mathrm{Me})$ Phe. Bioorg Med Chem Lett 2:453

Rando RR (1974) Irreversible inhibition of aspartate aminotransferase by 2-amino-3-butenoic acid. Biochemistry 13:3859

Rutjes FPJT, Wolf B, Schoemaker HE (2000) Applications of aliphatic unsaturated non-proteinogenic $\alpha-\mathrm{H}-\alpha$-amino acids. J Chem Soc Perkin Trans 1:4197-4212

Sawyer K (1997) Structure-based drug design: disease, targets, techniques and development, Veerapandian P (Ed), Marcel Dekker, New York, pp. 559

Shchetnikov GT, Zotova MA, Bruneau C, Dixneuf P, Osipov SN (2010) Synthesis of $\alpha$-alkynyl- $\beta, \beta, \beta$-trifluoroalanine derivatives by sonogashira cross-coupling reaction. Eur J Org Chem 2010:1587-1592

Shiau TP, Houchin A, Nair S, Xu P, Low E, Najafi R, Jain R (2009) Stieglitz rearrangement of $N, N$-dichloro- $\beta \beta$-disubstituted taurines under mild aqueous conditions. Biorg Med Chem Lett 19:1110-1114

Tanaka M (2007) Design and synthesis of chiral $\alpha, \alpha$-disubstituted amino acids and conformational study of their oligopeptides. Chem Pharm Bull 55:349-358

Taub D, Patchett AA (1977) Syntheses of $\alpha$-ethynyl3,4-dihydroxyphenylalanine and $\alpha$-vinyl-3,4dihydroxyphenylalanine. Tetrahedron Lett 18(32):2745-2748

Tendler SJB, Threadgill MD, Tisdale MJ (1987) Structural studies on bioactive compounds. Part 7. The design and synthesis of $\alpha$-substituted serines as prospective inhibitors of serine hydroxymethyltransferase. J Chem Soc Perkin Trans 1:2617-2623

Tolman V, Sedmera P (1988) Unsaturated amino acids: synthesis of trans-3,4-didehydro analogues of L-ornithine and L-arginine. Tetrahedron Lett 29:6183

Venkataiah M, Reddipalli G, Jasti L, Fadnavis N (2011) Chemoenzymatic synthesis of the azasugars 1,4-dideoxyallonojirimycin and 1,4-dideoxymannojirimycin. Tetrahedron Asymmetry $22: 1855-1860$

Venkatraman J, Shankaramma SC, Balaram P (2001) Design of folded peptides. Chem Rev 101(10):3131

Walsh C (1979) Enzymatic reaction mechanisms. W. H. Freeman, San Francisco 